\title{
Tier-scalable reconnaissance: the challenge of sensor optimization, sensor deployment, sensor fusion, and sensor interoperability
}

Wolfgang Fink, Thomas George, Mark A Tarbell

Wolfgang Fink, Thomas George, Mark A Tarbell, "Tier-scalable reconnaissance: the challenge of sensor optimization, sensor deployment, sensor fusion, and sensor interoperability," Proc. SPIE 6556, Micro (MEMS) and Nanotechnologies for Defense and Security, 655611 (3 May 2007); doi: $10.1117 / 12.721486$

SPIE Event: Defense and Security Symposium, 2007, Orlando, Florida, United States 


\title{
Tier-Scalable Reconnaissance: The Challenge of Sensor Optimization, Sensor Deployment, Sensor Fusion, and Sensor Interoperability
}

\author{
Wolfgang Fink*a ${ }^{* a}$ Thomas George ${ }^{\mathrm{b}}$, Mark A. Tarbell ${ }^{\mathrm{a}}$ \\ ${ }^{a}$ California Institute of Technology, Visual and Autonomous Exploration Systems Research \\ Laboratory, Division of Physics, Mathematics \& Astronomy, 1200 E California Blvd, \\ Mail Code 103-33, Pasadena, CA 91125, USA; \\ ${ }^{b}$ ViaLogy LLC, 2400 Lincoln Ave, Altadena, CA 91001, USA
}

\begin{abstract}
Robotic reconnaissance operations are called for in extreme environments, not only those such as space, including planetary atmospheres, surfaces, and subsurfaces, but also in potentially hazardous or inaccessible operational areas on Earth, such as mine fields, battlefield environments, enemy occupied territories, terrorist infiltrated environments, or areas that have been exposed to biochemical agents or radiation. Real time reconnaissance enables the identification and characterization of transient events. A fundamentally new mission concept for tier-scalable reconnaissance of operational areas, originated by Fink et al., is aimed at replacing the engineering and safety constrained mission designs of the past. The tier-scalable paradigm integrates multi-tier (orbit $\Leftrightarrow$ atmosphere $\Leftrightarrow$ surface/subsurface) and multi-agent (satellite $\Leftrightarrow U A V / b l i m p \Leftrightarrow$ surface/subsurface sensing platforms) hierarchical mission architectures, introducing not only mission redundancy and safety, but also enabling and optimizing intelligent, less constrained, and distributed reconnaissance in real time. Given the mass, size, and power constraints faced by such a multi-platform approach, this is an ideal application scenario for a diverse set of MEMS sensors. To support such mission architectures, a high degree of operational autonomy is required. Essential elements of such operational autonomy are: (1) automatic mapping of an operational area from different vantage points (including vehicle health monitoring); (2) automatic feature extraction and target/region-of-interest identification within the mapped operational area; and (3) automatic target prioritization for close-up examination. These requirements imply the optimal deployment of MEMS sensors and sensor platforms, sensor fusion, and sensor interoperability.
\end{abstract}

Keywords: Tier-scalable reconnaissance, space exploration, hazardous environment, mission autonomy, integrated vehicle health management, multi-parameter optimization, design/performance optimization, (MEMS) sensors, sensor fusion, sensor interoperability

\section{INTRODUCTION}

Extra-terrestrial space exploration has created the need for robotic reconnaissance operations in extreme environments such as planetary atmospheres, surfaces, and subsurfaces. Robotic reconnaissance is needed as well in potentially hazardous or inaccessible operational areas on Earth, such as those related to military or terrorist activities, or areas that have been exposed to biochemical agents, radiation, or natural disasters. Real time reconnaissance enables the identification and characterization of transient events. A recently originated tier-scalable paradigm [1-8], incorporating multi-tier (orbit $\Leftrightarrow$ atmosphere $\Leftrightarrow$ surface/subsurface) and multi-agent (satellite $\Leftrightarrow U A V / b l i m p \Leftrightarrow$ surface/subsurface sensing platforms) hierarchical mission architectures, is required for the next generation of missions, paving the way for human exploration of the Moon and Mars, as well as for Earth-bound robotic reconnaissance operations.

Conventional robotic planetary exploration scenarios have favored single lander/rover missions, being driven primarily by safety and engineering constraints, at the expense of mission redundancy and science return. Although rovers are generally capable mobile platforms, they are incapable of exploring multiple sites on a planetary surface that are separated from each other by distances of $100 \mathrm{~km}$ or more (a crucial feature for a geologist). Rovers are also unlikely to explore potentially hazardous but scientifically interesting surface and subsurface terrains, which have great potential to yield significant geologic, geomorphologic, pedologic, paleohydrologic, climatic, and possibly exobiologic information.

*wfink@autonomy.caltech.edu; phone: +1-626-395-4587; website: http://autonomy.caltech.edu 
At the other extreme, orbiting satellites traditionally return immense global surveillance datasets, while often overlooking detailed information of local and regional significance. With the advent of the latest generation of orbiter missions, such as the ESA Mars Express and NASA Mars Reconnaissance Orbiter (MRO), high resolution optical instruments are now employed, such as the High Resolution Stereo Camera (HRSC) on Mars Express, and the High Resolution Imaging Science Experiment (HiRISE) on MRO. However, complete planetary surface coverage with these instruments is neither intended nor feasible, and follow-up investigations of particular regions of interest at high resolution are subject to the constraints of the satellite orbits, making monitoring of transient events difficult, if not altogether impossible.

In this respect, tier-scalable reconnaissance [1-8] represents a fundamentally new planetary exploration mission concept. Tier-scalable reconnaissance is aimed at replacing the engineering and safety constrained mission designs of the past. As described above, the tier-scalable paradigm integrates multi-tier (orbit $\Leftrightarrow$ atmosphere $\Leftrightarrow$ surface/subsurface) and multi-platform (satellite $\Leftrightarrow$ blimps $\Leftrightarrow$ ground agents/sensors) hierarchical mission architectures. Tier scalability not only introduces mission redundancy and safety, but enables distributed, science-driven, and less constrained, real-time reconnaissance simultaneously on global, regional, and local scales for prime locations on Earth, Mars, Titan, Venus, Europa, Ganymede, and other planetary bodies. Tier scalability allows for significantly increased science return, differing from conventional missions in yet another fundamental way: the paradigm necessitates fully autonomous robotic missions.

As with all space missions, payload mass, size, and power consumption remain the overarching constraints. Thus, to achieve the significantly higher benefits afforded by the tier-scalable reconnaissance architecture, the individual systems that make up the architecture ideally must themselves be miniaturized, if the overall mission payload is to remain within the range of current flagship missions. In fact, the miniaturization requirement may flow down to the component level, necessitating the extensive use of Micro Electro Mechanical Systems (MEMS). In addition to MEMS sensors and actuators, a novel, scalable approach to the interconnection of these MEMS devices is required to fully exploit the extensive capabilities of these devices. An internet-protocol (IP) based sensor fusion architecture based on interoperable sensors controlled by a "sensor policy manager" (SPM) is being proposed to manage the outputs of these MEMS networks. The diversity of sensors controlled by SPM allows it to autonomously and precisely identify planetary phenomena (measured by the sensors) and initiate proper actions in response.

\section{TIER-SCALABLE RECONNAISSANCE: OPERATIONAL CONTROL, AND TECHNICAL CONSIDERATIONS}

A typical operations scenario for a tier-scalable reconnaissance system is illustrated schematically in Fig. 1, which utilizes only 3 of a possible $n$ tiers. Multi-tiered, multi-agent, hierarchical, integrated mission architectures allow for varying degrees of independence from human intervention, while also permitting manual override at any level. For example, a human operator may communicate with the orbiter(s) directly. Or, he may choose to command the airborne units via the orbiters. Or, in so doing, he may choose to also command the ground-level reconnaissance agents via the airborne units. Conversely, a highly automated operation mode may be used, enabling autonomous reconnaissance missions as they are necessary, such as when the communication time lag prohibits meaningful teleoperation, or, e.g., on the rear side of the Moon.

The often overstated and misunderstood term autonomy is defined in the context of this article as the high-level automation of planetary reconnaissance missions, including automated data acquisition, data feature extraction, data analysis, identification of targets, goal prioritization, execution of goals, navigation, and guidance. As such, most currently deployed agents are not truly autonomous (with the exception for very basic and local obstacle avoidance) as they are, for the most part, Earth-commanded and teleoperated.

In the highly automated scenario, the satellites command and control the airborne agents autonomously, and the airborne agents in turn autonomously command and control the ground-tier reconnaissance agents (Fig. 1). This system integrates satellites with balloons/blimps (airships) and ground-tier agents (rovers, fixed landers, and other sensor platforms). The airborne and ground-tier agents can be sufficiently inexpensive (in terms of mass, size, power, and operational resources) to allow for the deployment of numerous, expendable agents that, collectively, can address specific science-driven questions within the operational areas of particular interest. Examples of such "inexpensive" agents are MEMS-based sensor platforms, and mini-rovers akin to Minerva, the lander/mini-rover on the Japanese asteroid sample-return mission 
Hayabusa. Multiple ground-tier and airborne agents can collectively explore the same science target(s) with complementary suites of instruments.

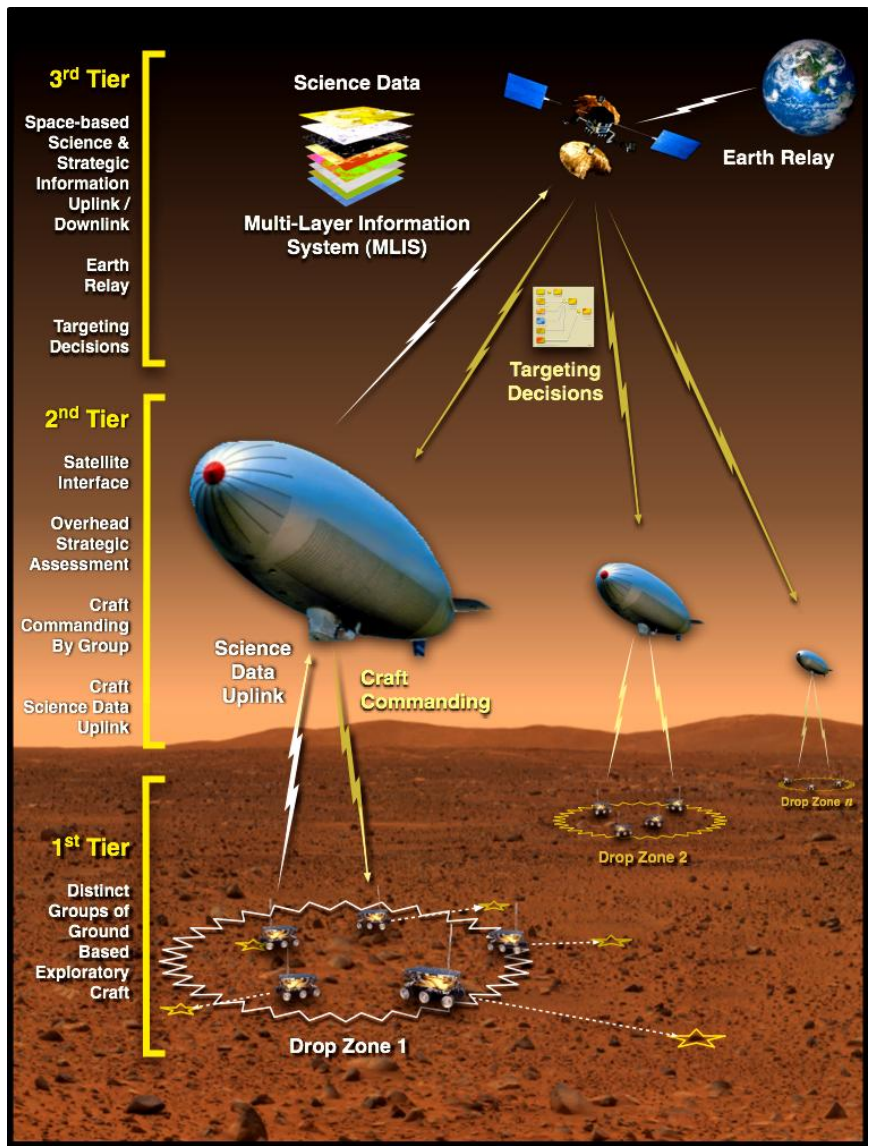

Fig. 1. Tri-level hierarchical multi-agent architecture for autonomous remote planetary exploration (after [1-7]).

To support and control such tier-scalable reconnaissance mission architectures, a high degree of operational autonomy is required. Essential requirements of such operational autonomy are:

1. Automatic mapping of an operational area from different vantage points (i.e., space, airborne, surface/subsurface).

2. Automatic feature extraction and target/region-of-interest identification within the mapped operational area (e.g., with software packages such as the Automated Geologic Field Analyzer (AGFA) [9]).

3. Automatic target prioritization for close-up examination (e.g., [9-13]), primarily by ground-tier agents, based on preliminary data, gathered in transit, and potentially linked to existing information from previous missions.

These requirements imply the optimal deployment of sensor platforms and individual sensors, sensor fusion, and sensor interoperability. Multiple prioritization scenarios can be conceived to evaluate the importance of individual targets or combinations of targets to be further examined during reconnaissance missions, which differ in their respective level of complexity. These scenarios can range from simple feature-based or feature-clustering-based prioritization [14-16] to prioritization via context-based clustering [17].

Recently, more advanced prioritization frameworks [10] have been developed using previously acquired, coarse feature/reconnaissance data that was pre-clustered using either general purpose clustering algorithms [14-16]) or clustering algorithms associated with special-purpose models [17]. This advanced class of algorithms facilitates (1) the selection of single or multiple targets, and (2) the selection of instruments used for the close-up examination of these targets in an operational area for potential information gain about the operational area. 
Tier-scalable reconnaissance mission architectures require not only a high level of operation autonomy [9-13], but also a new class of multi-purpose and reconfigurable instruments/sensors (e.g., intelligently reconfigurable snapshot hyperspectral imager [18, 19], and digital cameras with adapter-based microscopic and wide-angle imaging capability [20]) to render future missions more cost-effective (i.e., in terms of payload mass, size, and power requirements).

\section{MEMS: AN IDEAL CANDIDATE FOR TIER-SCALABLE RECONNAISSANCE}

Since its advent in the late 1980s, MEMS development has progressed by leaps and bounds and now encompasses a diverse range of sensors and actuators [21]. MEMS devices, due to their inherently low mass, size, and power, are ideal for both space applications as well as sensor network applications. A number of MEMS devices have been specifically developed for the purpose of robotic planetary exploration [22, 23].

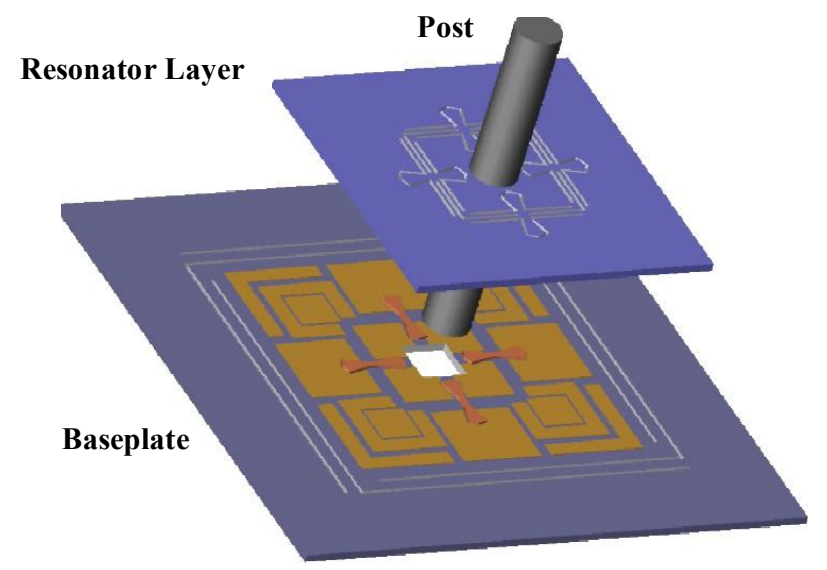

Fig. 2. Schematic, exploded view of a single-axis gyroscope developed at NASA's Jet Propulsion Laboratory (JPL) [23]. The inertial sensing element is the central post, about whose axis the rotation is sensed. The post is mounted on a layer containing in-plane orthogonal resonators. The post/resonator assembly is suspended over a substrate containing an arrangement of multiple electrodes for actuation, sensing and tuning the frequencies of the resonance modes. The gyroscope operates by "rocking" the post about an in-plane axis and consequently sensing the deflection about the orthogonal in-plane axis generated by the Coriolis force. The novel design is aimed both at closely matching the orthogonal resonance frequencies, and at maximizing the quality factor by minimizing mechanical coupling losses to the frame. The ultimate performance of the gyroscope has a strong dependence on both of these above factors.

MEMS technologies have been developed for a diverse set of applications. In this article we briefly survey a few sensor technologies and their applications for space exploration. For space exploration purposes, MEMS sensors can be categorized under two classes: physical sensors and chemical sensors. The early successes in MEMS technology were in the area of physical sensors, and more specifically in sensing the motion of a suspended proof mass for acceleration [24], rotation (e.g., MEMS micro-gyroscope, Fig. 2) [25, 23], and vibration measurements [26, 27]. Another early application was the measurement of ambient pressure, which was achieved by monitoring the deflection of microfabricated membranes [28]. Both pressure sensors and inertial measurement sensors have since become the "success stories" for MEMS and are now being offered commercially by a number of vendors. For in-situ planetary exploration applications, other physical measurements such as seismometry, temperature, and humidity measurements are also important. MEMSbased seismometers are essentially accelerometers with very low frequencies for their resonant elements [29, 30]. Temperature and humidity sensors could be used for instance in astrobiological investigations for studying potential moisture rich areas such as on canyon floors on Mars as well as dark/bright slope streaks within martian craters (to determine brines versus dust avalanches as the cause). MEMS devices have been demonstrated that can simultaneously measure both the temperature and the humidity [31].

A number of MEMS-based technologies exist in the arena of chemical sensors. For planetary exploration purposes, chemical sensors are required for detecting and characterizing compounds in solid, liquid, and gaseous forms. For instance, in the case of gas detection miniaturized methane sensors have been developed $[32,33]$ that could be utilized for the detection of methane outgassing on Mars and Titan. Miniature gas chromatographs $[34,35]$ have been developed 
that could potentially be coupled to miniature mass spectrometers (Fig. 3) [22, 36]. "Electronic Nose" instruments based on miniaturized chemi-resistors or chem-FETs (Field Effect Transistors) [37] have also been developed.

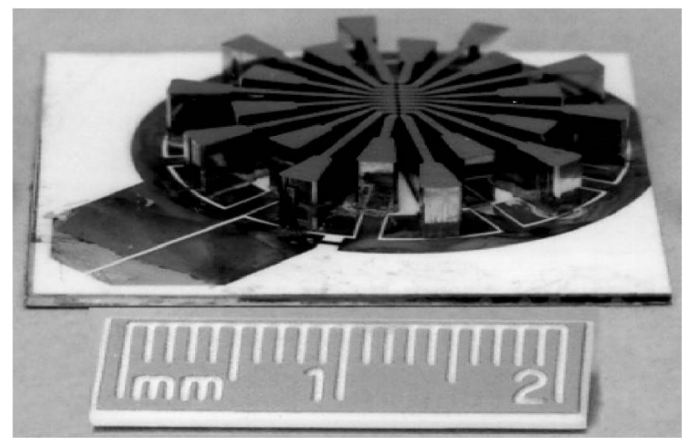

Fig. 3. A prototype MEMS-fabricated quadrupole mass filter for a miniature GCMS (Gas Chromatograph Mass Spectrometer) system [22].

For the sensing of liquid species, the pre-eminent technique is Nuclear Magnetic Resonance (NMR) spectroscopy. Recently, a miniaturized Force Detected Nuclear Magnetic Resonance (FDNMR) spectrometer was demonstrated for the detection of water and organic materials [38]. In the case of solid-state chemical analysis, Fig. 4 shows a novel, MEMSenabled Atmospheric Electron X-ray Spectrometer (AEXS) that is capable of rapid elemental analysis in the atmospheric ambient [38]. The AEXS consists of a miniature high-voltage electron source that is vacuum-encapsulated by a MEMSfabricated SiN membrane. The 200-nm-thick membrane allows the generated electron beam to traverse through it and excite characteristic $\mathrm{x}$-ray fluorescence from planetary materials.

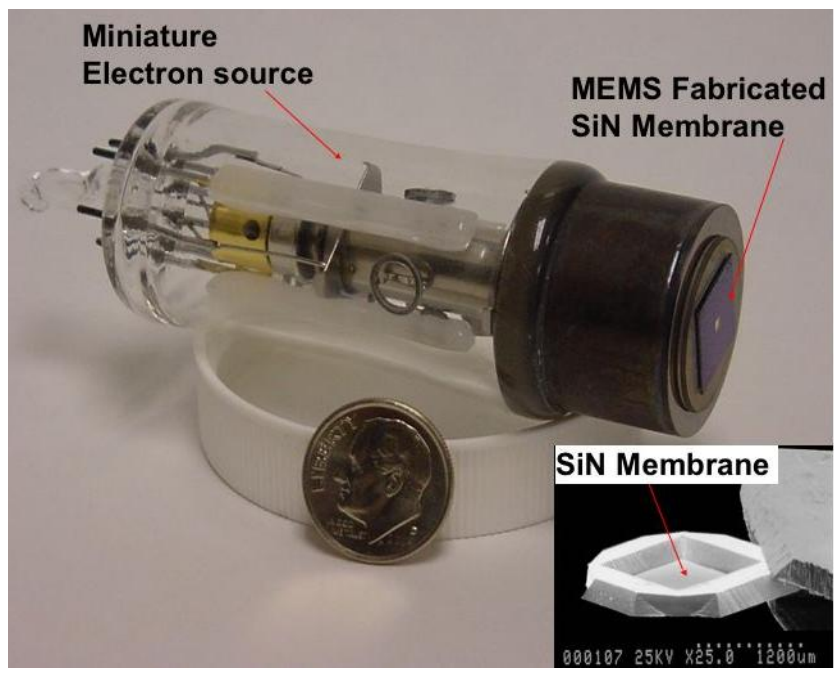

Fig. 4. MEMS-based vacuum encapsulated miniature electron source [38]. Inset shows the MEMS-fabricated 200nm-thick $\mathrm{SiN}$ encapsulation membrane that is anodically bonded to the electron source. The miniature electron source is the primary component of an Atmospheric Electron X-ray Spectrometer developed for rapid chemical identification of planetary materials by the analysis of electron-beam excited x-ray fluorescence.

Despite the diversity of MEMS-based technologies, three major challenges stand in the way of realizing the ultimate vision of deploying MEMS sensor networks in tier-scalable reconnaissance architectures:

1. Fully autonomous, event-based processing of MEMS sensor outputs, and rapid communication to appropriate consumers of sensor information.

2. Rapid analysis of orthogonal sensor inputs to precisely identify events of interest with low false positives and false negatives.

3. Robust detection of weak signals generated by each individual MEMS sensor in high clutter environments. 
To date, MEMS development has largely focused on the production of discrete devices, with only recent interest in MEMS-based sensor networks. Gulati and co-workers [39] have developed a novel IP v.6-based "Sensor Policy Management" (SPM $\left.{ }^{\mathrm{TM}}\right)$ product that delivers IP-based interoperability for practically all types of diverse MEMS sensors. SPM ${ }^{\mathrm{TM}}$ embodies within it a sensor-fusion engine that can analyze the inputs from multiple, orthogonal sensors in order to better identify the events of interest and reduce the numbers of false positives and false negatives. Novel sensor fusion algorithms have been developed [39] to rapidly reduce the multi-dimensional sensor output datasets to unique identifiers of events of interest.

Finally, the primary downside to the miniaturization of sensors is the significant reduction in signal-to-noise ratio (SNR) for MEMS sensors (Fig. 5). Advanced signal processing algorithms are required for extracting weak signals from noisy backgrounds. George [40] describes a class of "active signal processing" algorithms that have been successfully applied to the detection and quantitation of weak fluorescence signals from the background clutter in genomic biochips. Also, using such algorithms a $>10 \mathrm{x}$ improvement was achieved in the rotation rate bias stability for a MEMS Gyroscope.

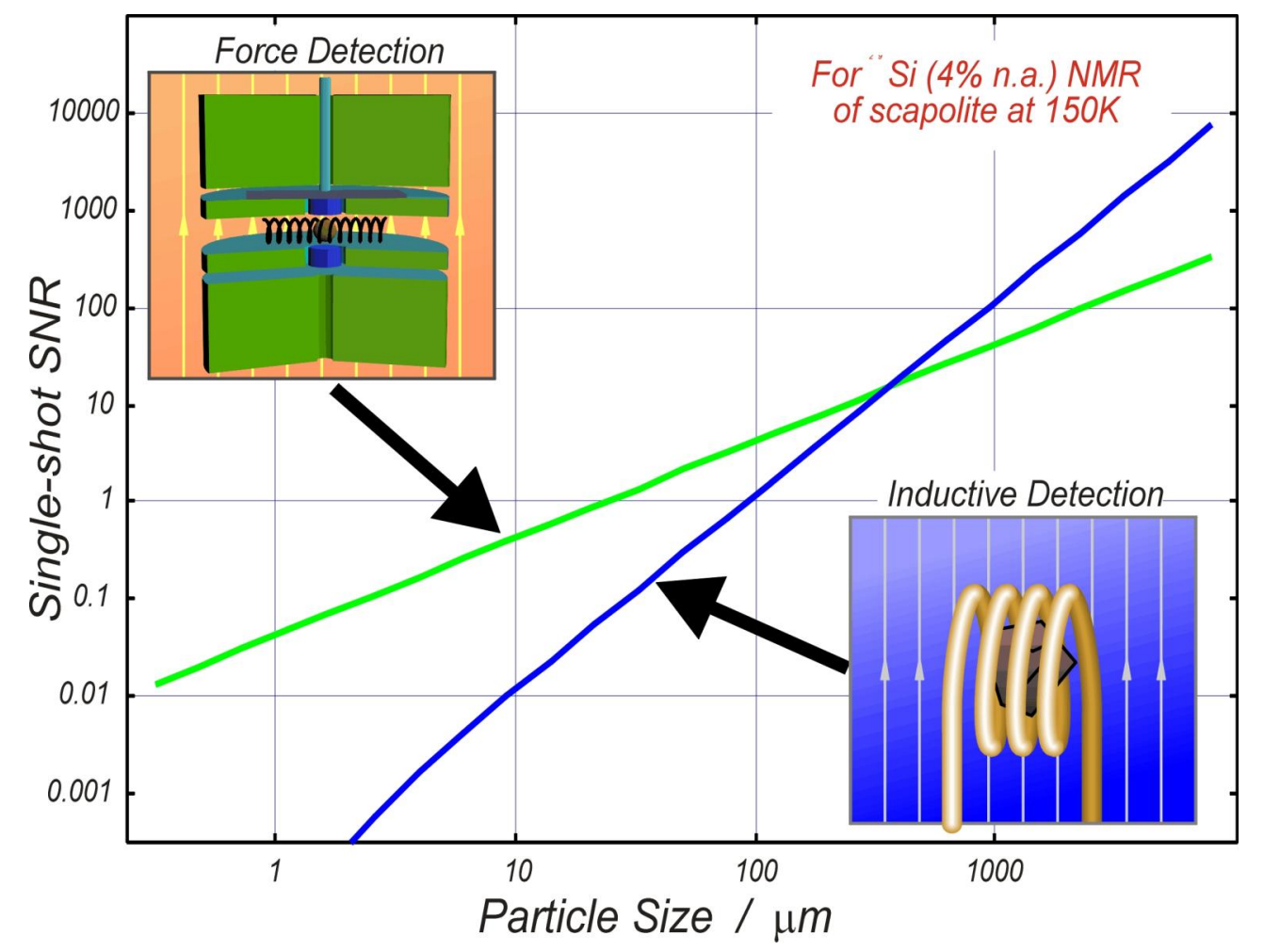

Fig. 5. Comparison of theoretically achievable signal-to-noise ratios (SNRs) for two modes of observing Nuclear Magnetic Resonance (NMR) spectroscopy in materials. The conventional, inductive detection technique relies on the generation of an induced current in the detector coil due to the motion of the sample's nuclear spin magnetic moment. An alternative force-detected NMR (FDNMR) spectroscopy is being developed in which a detector magnet responds to the motion of the sample's nuclear magnetic moment at the resonance frequency of a microfabricated cantilever [40]. Although both techniques are amenable to miniaturization, the SNR decreases with the characteristic dimension, in this case represented by the size of the sample being observed. At MEMS sizes, although the FDNMR technique has superior SNR in comparison to the conventional technique, the sensitivity is still quite small and requires advanced signal processing techniques in order to realize the promise of this new technology.

\section{APPLICATIONS OF TIER-SCALABLE RECONNAISSANCE MISSION ARCHITECTURES}

Integrated multi-tier, multi-agent, hierarchical mission architectures, potentially equipped with MEMS-based technologies, would overcome the inherent challenge of geologic planetary surface exploration [1-8]: Airborne agents 
(orbiters in conjunction with balloons/blimps) possess overhead perspectives at different length scales/resolutions, which could provide guidance and control to ground-based agents (e.g., mobile rover units). Such airborne agents would map out operational areas of interest (e.g., prime sites, such as dark/bright slope streaks within a crater, or "hot" zones within a battlefield) and acquire terrain images that subsequently could be processed through automated feature-extraction software packages, such as the Automated Geologic Field Analyzer (AGFA) [9]. The feature data would be analyzed by science prioritization algorithms (e.g., [9-13]) to choose potential targets for close examination by subsequently deployed (MEMS) sensors or rovers and for determining safe passages to their designated targets. At the respective targets, the rovers would conduct in-situ science experiments using their (MEMS) sensor payload and thereby gather data that complement the remote sensing data obtained by the airborne units. In addition, such a system could help direct rovers equipped with drills for in-situ subsurface sampling, or, from a military/surveillance point of view, could help direct rovers to deliver payloads (e.g., explosives) or remove hazardous obstacles (e.g., landmines) [41].

\subsection{Planetary Exploration Applications}

Tier-scalable reconnaissance missions not only introduce redundancy, and thus unprecedented mission reliability and safety, they also enable spanning larger surface areas than previously possible - mimicking the way geologists explore regions on Earth - and thereby allow for increased science return. Several example scenarios are outlined below [1]:

- Planetary bodies with atmospheres \& non-extreme surface temperatures (e.g., Earth and Mars): Orbiter-guided deployment and control of balloon/blimp units, which in turn deploy and control both mobile and immobile ground-based agents (3 tiers).

- Planetary bodies with atmospheres \& extreme surface temperatures (e.g., Venus and Titan): Orbiter-guided deployment of balloon/blimp units. If surface temperatures permit, deployment and communication can be achieved with temperature-resistant immobile sensor webs or mobile agents (2-3 tiers).

- Planetary bodies w/o atmospheres \& extreme surface conditions (e.g., Mercury, Moon, Europa): Orbiterguided deployment of and communication with ground-based mobile agents and immobile sensor webs (2 tiers).

Non-traditional autonomous missions to remote planetary bodies will be necessary [1-7, 42-46] primarily to allow intelligent and less constrained access to scientifically interesting targets on planetary bodies of the Solar System not currently feasible with conventional mission designs, in locations such as: (1) canyons, (2) mountain ranges, (3) chaotic and rugged terrain, (4) impact basins/craters, (5) volcanoes, and (6) liquid pools or lakes. These and many other locales of scientific interest on the planetary bodies of the Solar System are particularly crucial for astrobiologic-oriented exploration in general [45-47], and sample return missions in particular [42, 43].

\subsection{Terrestrial Applications}

Potential terrestrial applications range from Homeland Security, border control, (battlefield) surveillance, telecommunications relay, and weather observations, to monitoring of military checkpoints, standoff detection and delivery/removal of explosives [41] (e.g., landmine clean-up). This opens the door to robotic reconnaissance or clean-up operations in potentially hazardous or inaccessible environments such as those related to military or terrorist activities, or areas that have been exposed to biochemical agents, radiation, or natural disasters.

As one example, DARPA has initiated the "Integrated Sensor Is Structure" (ISIS) program that "is developing a stratospheric airship with sensor antennas that will include a radar nearly as large as the airship. This would create a battlefield surveillance platform with extreme endurance, and equally extreme resolution for its air and battlefield scans via radar and other carried sensors. This project is associated with Lockheed's High Altitude Airship program, which is intended to soar at over 65,000 feet for over a month at a time, and could also play a significant role in ballistic missile and cruise missile defense." (excerpt from Defense Industry Daily News Release from June 09, 2006 [48]; for DARPA ISIS program see [49] and for Lockheed Martin High Altitude Airship (HAA) program see [50, 51]).

The tier-scalable reconnaissance paradigm can comprise anywhere from 1 to $n$ tiers. For example, in addition to the spaceborne and ground tiers, there may be several airborne tiers deployed, such as airplanes (e.g., AWACS (Airborne Warning and Control System)) and UAVs (e.g., drones) at different altitudes. Furthermore, the tier-scalable reconnaissance paradigm is not limited to space/aerial/ground applications but can be equally applied to watersurface/water-subsurface/ocean-floor scenarios both for military and commercial purposes (e.g., detection and harvesting of ocean-floor resources such as polymetallic/manganese nodules, or methane clathrates, etc.). 


\section{IMPLEMENTATION OF A TEST BED FOR TIER-SCALABLE RECONNAISSANCE}

\subsection{Indoor Miniature Test Bed}

As a first step towards a test bed for tier-scalable reconnaissance, we (WF and MAT) have developed an indoor miniature test bed consisting of a 4' x 5' operational area with a mast-mounted camera "hovering" above, emulating the perspective of an airborne platform (Fig. 6). Using building blocks of differing size, color, albedo, and shape, rocks with a certain feature space were simulated. Fully maneuverable mini-rover units, equipped with onboard optical cameras, were autonomously commanded via a remote wireless tele-commanding and control system to "interesting" science targets determined by a feature extraction and science goal prioritization software package (AGFA [9]), using the overhead camera perspective.

The underlying integrated software system for the miniature test bed comprised the following software components:

- Overhead image capture

- Image processing for target identification with AGFA [9] (image segmentation, etc.)

- Overhead tracking and navigation for ground-based rovers

- Feature extraction of identified targets with AGFA [9]

- Field site classification and science goal prioritization with AGFA [9]

- Path planning for ground-based rovers

- Wireless commanding interface of ground-based rovers.
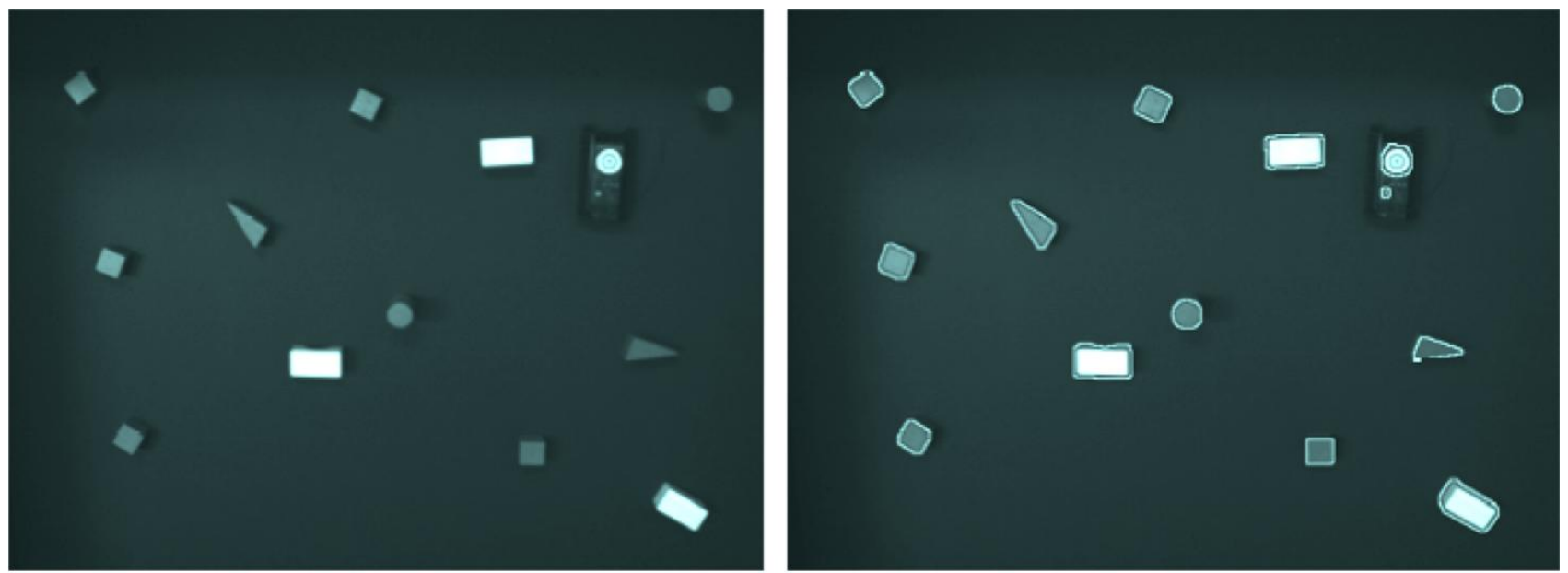

Fig. 6. Left: overhead view of miniature test bed for air-guided autonomous surface exploration with ground-based rover unit; right: image processing via AGFA [9] of science targets within the operational area.

This miniature test bed successfully proved the concept of tier-scalable reconnaissance by demonstrating closed-loop (autonomous, i.e., no human in the loop) deployments (i.e., commanding and navigation) of the mini-rover to multiple (up to seven) science targets within the operational area without collisions, exclusively guided and commanded by the overhead perspective.

The developed hard- and software platform allowed for:

- Testing of algorithms for automated geologic field site classification and science goal prioritization

- Testing of algorithms for navigation of ground-based science craft

- Testing of algorithms for path planning 
- Testing of algorithms for operating multiple science craft simultaneously within a field site for science-driven exploration in parallel

- Testing of algorithms for closed-loop (autonomous, i.e., no human in the loop) science context driven exploration with ground-based agents.

\subsection{Outdoor Test Bed}

To study and validate the tier-scalable reconnaissance concept under more realistic (outdoor) conditions than is possible with the above miniature test bed, we (WF and MAT) have begun development and implementation of an outdoor test bed consisting of remote controllable robotic platforms (via wireless Ethernet) and blimps [52].

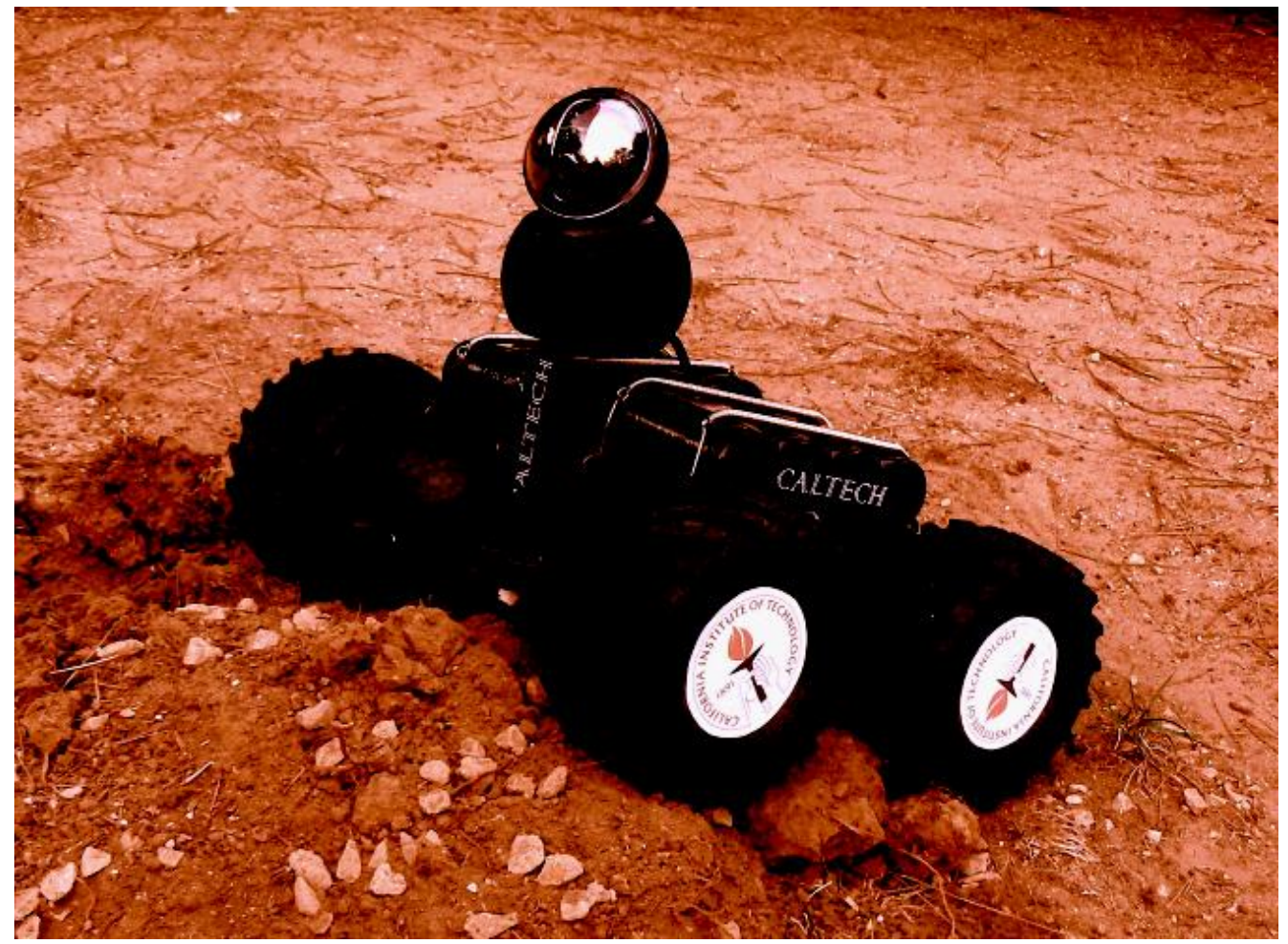

Fig. 7. 4WD remote controllable robotic platform as a representative mobile ground-tier agent of the tier-scalable reconnaissance mission test bed [52].

In particular, a 4WD remote controllable robotic platform is utilized as a representative mobile ground-tier agent (Fig. 7). To enable remote user control of the ground-tier agent, a network TCP/IP connection is first established between the CPU aboard the mobile platform (via its wireless LAN) and the computer hosting the front-end control software.

Once this connection is established, the mobile platform is able to transmit video frames and (MEMS) sensor data in a packetized and compressed format. The mobile platform also transmits its housekeeping data (battery level, $\mathrm{h} / \mathrm{w}$ sensor data, etc.) and awaits sensor and drive commands from the front-end software. The video and sensor data are treated similarly, however, the video data first are preprocessed into a suitable data format. This is accomplished by packetizing the video. Each non-interlaced stream frame of video data is compressed and inserted into a packet, tagging the data as to type, length, timestamp, and sequence. This has the advantage over time-division multiplexing of allowing for realtime synchronization to occur on the receiving end with minimal reconstruction processing. The network connection is thus used as a virtual N-receiver broadcast channel, each channel being a Q-ary data channel, providing the same mechanism for video, (MEMS) sensor, or hardware housekeeping data.

For the purpose of commanding the ground-tier agent interactively (later autonomously), the front-end software has an integrated video panel (Fig. 8) for displaying the transmitted video frames from the mobile platform's on-board camera (Fig. 7); it is also outfitted with a USB-based joystick device. The user's movements of the joystick are translated into camera orientation and wheel rotation commands, and are sent to the mobile platform. As the mobile platform begins to 
move, it also sends back video, sensor, and housekeeping data, which are displayed on the front-end. With this feedback information, a user (or automated control software for autonomous operation) is able to control the ground-tier agents interactively (or automatically) from anywhere in the world, in near real-time.

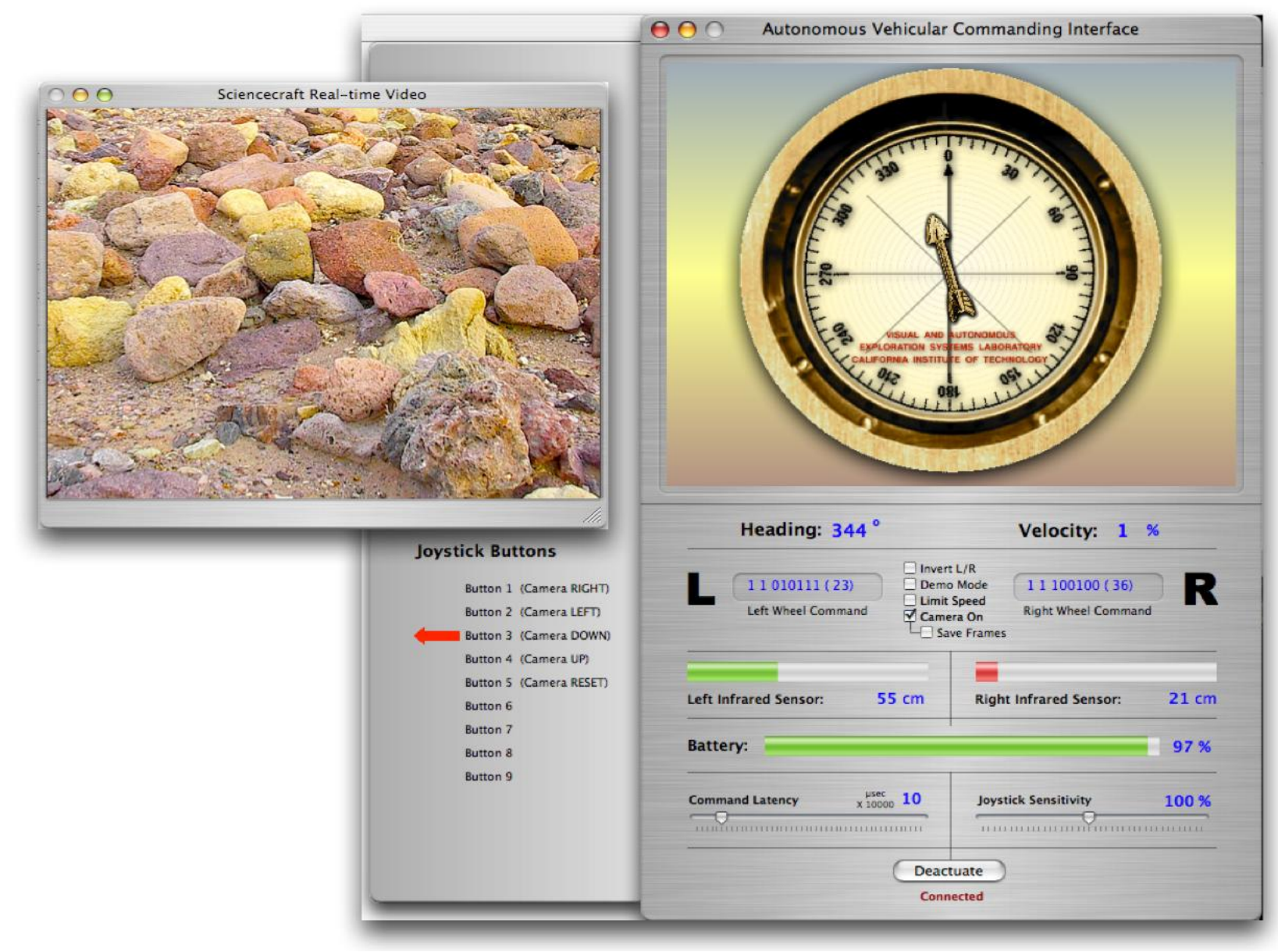

Fig. 8. Autonomous Vehicular Commanding Interface, controlling a 4WD remote robotic platform in near real-time [52].

Figure 8 shows the Autonomous Vehicular Commanding Interface ("software front-end") commanding the 4WD remote robotic platform ("agent") pictured in Fig. 7. When the software front-end is networked with the agents, they are linked in a near-real-time positive feedback loop: A user controlling the joystick sends drive and sensor commands to the active agent; the agent executes the commands, and replies with data and video sent back to the user.

The user, controlling the joystick, is in the loop for the purpose of the development of automated software and algorithms to control the remote agents. Once developed, such automated software can be "plugged in" in lieu of the user to automatically control the agents. Manual user override is always available, however.

\subsection{Implications and Outlook for Test Bed Development}

Individual components of the tier-scalable mission architecture proposed by Fink et al. [1-8] are either under development or have already been tested and proven "in the field". These include orbiters, balloons/blimps/airships, and ground-based agents such as rovers and landers as well as immobile sensor webs. The biggest challenge, however, appears to be not so much the hardware but the "intelligent" software that would enable all the components of a multitier multi-agent mission to be integrated and function autonomously.

A ground-tier test bed by itself already enables the development, implementation, field-testing, and validation of exploration/navigation, feature extraction (e.g., [9]), and science goal prioritization (e.g., [9-13]) algorithms/software and strategies. Furthermore, the ground-tier agents may act as ideal (mobile) platforms for field-testing (novel) instruments 
(e.g., intelligently reconfigurable snapshot hyperspectral imager $[18,19]$ and digital cameras with adapter-based microscopic and wide-angle imaging capability [20]) and (MEMS) sensor equipment. With the near-term advent of the airborne tier, a full-fledged Earth-based test bed for tier-scalable reconnaissance will eventually become available (being developed, implemented, and field-tested by some of the authors), as some of the airborne agents can take on the role of the space-borne agents (e.g., orbiters) at higher altitudes above the operational area. This test bed will then allow the development, implementation, field-testing, and validation of software packages for both intra-tier and inter-tier operations, allowing all agents to communicate with one another and to navigate and explore operational areas with greatly reduced (and ultimately without) help from ground operators, thus affording more mission autonomy/flexibility and increased science return.

\section{DISCUSSION \& OUTLOOK}

Fully embracing the tier-scalable reconnaissance concept originated by Fink et al. [1-8], NASA recently has been soliciting proposals calling for technology development for multi-tier sensor webs: "Sensor webs of the future may include space-based, airborne, and in-situ sensors, all working together in a semi-closed loop system in which "smart" sensors sense what is happening per their designed sensing capabilities and feed that information into a control system. Based on the sensor inputs, the control system then modifies the environment (instrument pointing, data collection on or off, etc.) and causes the sensors to take in and provide new information to the control system." (excerpt from Science Mission Directorate NASA Research Announcement "Advanced Information Systems Technology" Solicitation: NNH05ZDA001N-AIST). Moreover, in testimony to Congress in May 2005, NASA Administrator Michael Griffin included the following statement: "In the future, NASA plans to develop a "sensor web" to provide timely, on-demand data and analysis to users who can enable practical benefits for scientific research, national policymaking, economic growth, natural hazard mitigation, and the exploration of other planets in this solar system and beyond."

Some of the major advantages afforded by tier-scalable reconnaissance architectures include: (1) Overhead navigation, enabling deployment of less smart, therefore less costly, expendable ground agents; (2) the capability to drop multiple expendable ground agents into regions of interest (e.g., "hot" zones); (3) mission safety and redundancy; (4) optimized target identification and obstacle avoidance; and (5) efficient commanding of multiple ground agents.

Potential onboard instruments (especially for the airborne tier) may include: (1) Ground penetrating radar (GPR); (2) optical, IR, UV cameras; (3) detectors for Improvised Explosive Devices (IED); (4) hyperspectral cameras; (5) electrochemical sniffing devices; and (6) other sensors, including the MEMS sensor suite discussed above.

Real time reconnaissance enables identification and characterization of transient events, both planetary and terrestrial, including detection and location of mobile laboratories for and transport of weapons and explosives. Tier-scalable reconnaissance not only introduces mission redundancy and safety, but enables and optimizes intelligent, less constrained, and distributed reconnaissance, detection, and subsequent in-situ sampling of targets of interest, including delivery of payloads or removal of hazards (e.g., [41]) within the operational area.

We envision that multi-tier, multi-agent, hierarchical mission architectures for remote planetary reconnaissance, as described here, are feasible within a timeframe of 10-15 years. Integrated orbiter-airship missions, especially suitable for the exploration of Titan, Venus, and Mars, are envisioned to be feasible within a decade from now. Tier-scalable reconnaissance as it applies to terrestrial applications, such as in Defense and Homeland Security, is being implemented at the time of this writing (e.g., DARPA ISIS program [49] and Lockheed Martin HAA program [50]).

\section{REFERENCES}

1. W. Fink, J.M. Dohm, M.A. Tarbell, T.M. Hare, V.R. Baker, "Next-Generation Robotic Planetary Reconnaissance Missions: A Paradigm Shift," Planetary and Space Science, 53, 1419-1426, 2005.

2. W. Fink, J.M. Dohm, M.A. Tarbell, T.M. Hare, V.R. Baker, "Next-Generation Robotic Planetary Surface/Subsurface Reconnaissance Missions: A Paradigm Shift" [abstract 1977], in $36^{\text {th }}$ Lunar and Planetary Science Conference Abstracts [CD-ROM], Lunar and Planetary Institute, Houston, 2005.

3. W. Fink, J.M. Dohm, M.A. Tarbell, T.M. Hare, V.R. Baker, "Next-Generation Robotic Planetary Reconnaissance Missions: A Paradigm Shift," Geochimica et Cosmochimica Acta, Volume 69, Number 10S, A533, 2005. 
4. W. Fink, J.M. Dohm, M.A. Tarbell, T.M. Hare, V.R. Baker, D. Schulze-Makuch, R. Furfaro, A.G. Fairén, T.P.A. Ferré, H. Miyamoto, G. Komatsu, W.C. Mahaney, "Multi-tier Multi-agent Autonomous Robotic Planetary Surface/Subsurface Reconnaissance For Life" [abstract 1433], in $37^{\text {th }}$ Lunar and Planetary Science Conference Abstracts [CD-ROM], Lunar and Planetary Institute, Houston, 2006.

5. W. Fink, J.M. Dohm, M.A. Tarbell, T.M. Hare, D. Schulze-Makuch, R. Furfaro, V.R. Baker, "Tier-Scalable Reconnaissance for Remote Planetary Exploration," abstract $25^{\text {th }}$ International Space Development Conference, Los Angeles, 2006.

6. W. Fink, J.M. Dohm, M.A. Tarbell, T.M. Hare, V.R. Baker, D. Schulze-Makuch, R. Furfaro, A.G. Fairén, T.P.A. Ferré, H. Miyamoto, G. Komatsu, W.C. Mahaney, "Autonomous Tier-Scalable Reconnaissance Missions For Remote Planetary Exploration," Proceedings of the $4^{\text {th }}$ International Planetary Probe Workshop, Pasadena, 2006.

7. W. Fink, J.M. Dohm, M.A. Tarbell, T.M. Hare, V.R. Baker, D. Schulze-Makuch, R. Furfaro, A.G. Fairén, T.P.A. Ferré, H. Miyamoto, G. Komatsu, W.C. Mahaney, "Tier-Scalable Reconnaissance Missions For The Autonomous Exploration Of Planetary Bodies," IEEE Aerospace Conference Proceedings, Big Sky, Montana, 2007.

8. Caltech's Visual and Autonomous Exploration Systems Research Laboratory Web site (by W. Fink) http://autonomy.caltech.edu/autonomy/tierscalable.html

9. W. Fink, A. Datta, V.R. Baker, "AGFA: (Airborne) Automated Geologic Field Analyzer," Geochimica et Cosmochimica Acta, Volume 69, Number 10S, A535, 2005.

10. W. Fink, "Generic Prioritization Framework for Target Selection and Instrument Usage for Reconnaissance Mission Autonomy," Proceedings of IEEE World Congress on Computational Intelligence (WCCI), Vancouver, Canada, 11116-11119, 2006.

11. R. Furfaro, J.M. Dohm, W. Fink, D. Schulze-Makuch, A.G. Fairén, M.A. Tarbell, T.M. Hare, V.R. Baker, "MultiLayer Fuzzy Logic-based Expert System for Conducting Tier-scalable Planetary Reconnaissance" [abstract 1257], in $37^{\text {th }}$ Lunar and Planetary Science Conference Abstracts [CD-ROM], Lunar and Planetary Institute, Houston, 2006.

12. R. Furfaro, J.M. Dohm, W. Fink, "Fuzzy Logic Expert System for Tier-scalable Planetary Reconnaissance," 9th International Conference on Space Operations, AIAA, Rome, Italy, June 19-23, 2006.

13. R. Furfaro, J.M. Dohm, W. Fink, "Autonomy in Planetary Exploration: Fuzzy Expert System for Tier-Scalable Reconnaissance," abstract $25^{\text {th }}$ International Space Development Conference, Los Angeles, 2006.

14. R.O. Duda, et al., Pattern Classification and Scene Analysis, John Wiley \& Sons, $2^{\text {nd }}$ edition, 2000.

15. C.M. Bishop, Neural Networks for Pattern Recognition, Clarendon Press, Oxford, 1995.

16. C.K.I. Williams, "An MCMC Approach to Hierarchical Mixture Modelling," Advances in Neural Information Processing Systems 12, S. A. Solla, T. K. Leen, K.-R. Mueller, eds., MIT Press, 2000.

17. W. Fink, R. Castano, A. Davies, E. Mjolsness, "Clustering Algorithm for Mutually Constraining Heterogeneous Features," Technical Report JPL-ICTR-01-5, 2001.

18. W.R. Johnson, D.W. Wilson, W. Fink, M. Humayun, G. Bearman, "Snapshot hyperspectral imaging in ophthalmology," Journal for Biomedical Optics Jan-Feb;12(1):014036, 2007.

19. G.H. Bearman, W.R. Johnson, W. Fink, D.W. Wilson, "An Intelligently Reconfigurable Snapshot Imaging Spectrometer For Planetary Exploration" [abstract 1103], in $38^{\text {th }}$ Lunar and Planetary Science Conference Abstracts [CD-ROM], Lunar and Planetary Institute, Houston, 2007.

20. W. Fink, W.C. Mahaney, K.R. Kuhlman, "Adapter-based Microscopic and Wide-angle Imaging Capability For Digital Cameras For Planetary Exploration and Astrobiology" [abstract 2397], in 37 ${ }^{\text {th }}$ Lunar and Planetary Science Conference Abstracts [CD-ROM], Lunar and Planetary Institute, Houston, 2007.

21. "The MEMS Handbook: MEMS Applications," ed. M. G. Hak, CRC Press, Florida, 2006, ISBN:0-8493-9139-3

22. T. George, "MEMS/NEMS development for Space Applications at NASA/JPL," Proc. SPIE, 4755, 556 (2002).

23. T. George, "Overview of MEMS/NEMS Technology Development for Space Applications at NASA/JPL," Proc. SPIE, 5116, 136 (2003).

24. N. Yazdi, F. Ayazi, and K. Najafi, "Micromachined inertial sensors," Proc. IEEE, 86, 1640 (1998).

25. M. Weinberg, J. Connelly, A. Kourepenis, and D. Sargent, "Microelectromechanical instrument and systems development at the Charles Stark Draper Laboratory, Inc," Proc. 16th DASC. AIAA/IEEE 2, 33 (1997).

26. A. Gupta, R. Singh, V.K. Jain, and V. Kumar, "Development of micromachined vibration sensors for MEMS applications," Proc. SPIE, 3903, 153 (1999).

27. E. Ollier, P. Philippe, C. Chabrol, and P. Mottier, "Micro-Opto-Mechanical Vibration Sensor Integrated on Silicon," J. Lightwave Tech., 17, 26 (1999).

28. K.E. Petersen, "Silicon as a mechanical material", Proc. IEEE, 70, 420 (1982). 
29. W. B. Banerdt and W. T. Pike, "A Miniaturized Seismometer For Subsurface Probing On Mars," Conf. Proc. Geophysical Detection of Subsurface Water on Mars, Lunar and Planetary Institute, 2001.

30. Y. Suzuki and Y-C. Tai, "Micromachined high-aspect-ratio parylene beam and its application to low-frequency seismometer," Proc. $16^{\text {th }}$ Annual International IEEE Conference on Micro Electro Mechanical Systems, 486 (2003).

31. Z. Fang, Z. Zhao, Y. Wu, B. Zhang and Y-Z. Wang, "Integrated temperature and humidity sensor based MEMS," Proc. IEEE Int. Conf. Information Acquisition, 84 (2004).

32. S. Sosin, C. Moldovan, and R. Iosub, " Design and simulations for a calorimetric micro-sensor for methane detection," Proc. Int. Semicond. Conf. CAS, 2, 381 (2004).

33. K.D. Mitzner, J. Sternhagen, and D.W. Galipeau, "Development of a micromachined hazardous gas sensor array," Sens. \& Actuat. B: Chem. 93, 92 (2003).

34. C-J Lu, W.H. Steinecker, W-C Tian, M.C. Oborny, J.M. Nichols, M. Agah, J.A. Potkay, H.K. L. Chan, J. Driscoll, R.D. Sacks, K.D. Wise, S.W. Pang and E.T. Zellers, "First-generation hybrid MEMS gas chromatograph," J. Roy. Soc. Chem. Lab. Chip 5, 1123 (2005).

35. E.S. Kolsear and R.R. Reston, "Review and Summary of a Silicon Micromachined Gas Chromatography System," IEEE Trans. Comp., Packag., \& Manuf. Tech., 66, 481(1998).

36. M. Geear, R.R.A. Syms, S. Wright and A.S. Holmes, "Monolithic MEMS quadrupole mass spectrometers by deep silicon etching", J. Microelectromech. Syst., 14, 1156 (2005).

37. J. Gong, Q. Chen, W. Fei, and S. Seal, "Micromachined nanocrystalline SnO2 chemical gas sensors for electronic nose", Sens. \& Actuat. B: Chem., 102, 117 (2004).

38. T. George, E. Urgiles, R. Toda, J.Z. Wilcox, S. Douglas, C-S. Lee, K. Son, D. Miller, N. Myung, L. Madsen, G. Leskowitz, R. El-Gammal, and D. Weitekamp, "MEMS-based Micro Instruments for In-Situ Planetary Exploration," Proc. SPIE, 5836, 20 (2005).

39. S. Gulati (private communication describing ViaLogy LLC products).

40. T. George, "Active Signal Processing: A counter-intuitive approach to enhancing Signal-to-Noise ratio via noise injection," Proc. Mat. Res. Soc., to be published (2007).

41. W. Fink and M.A. Tarbell, "Tier-scalable Reconnaissance for Detection and Removal of Explosives," invited poster presentation at the Combating Terrorism Technology Support Office Technical Support Working Group (TSWG) Explosives Detection Conference, Miami, Florida, June, 2006.

42. D. Schulze-Makuch, J.M. Dohm, A.G. Fairén, V.R. Baker, W. Fink, R.G. Strom, "Venus, Mars, and the Ices on Mercury and the Moon: Astrobiological Implications and Proposed Mission Designs," Astrobiol., 5, 778-795, 2005.

43. D. Schulze-Makuch, J.M. Dohm, A.G. Fairén, V.R. Baker, W. Fink, R.G. Strom, "Sample Return Missions to Mars, Venus, and the Ices on Mercury and the Moon" [abstract 1324], in 37th Lunar and Planetary Science Conference Abstracts [CD-ROM], Lunar and Planetary Institute, Houston, 2006.

44. J.M. Dohm, W. Fink, D. Schulze-Makuch, A.G. Fairén, V.R. Baker, R. Furfaro, M. Tarbell, T.M. Hare, "Tierscalable Reconnaissance To Test Overarching Geological Theories and Locate Prime Targets on Mars," abstract $25^{\text {th }}$ International Space Development Conference, Los Angeles, 2006.

45. D. Schulze-Makuch, J.M. Dohm, C. Fan, A.G. Fairén, J.A.P. Rodriguez, V.R. Baker, W. Fink, "Exploration of Hydrothermal Targets on Mars," Icarus (in press), 2007.

46. D. Schulze-Makuch, J.M. Dohm, A.G. Fairén, W. Fink, C. Fan, J.A.P. Rodriguez, V.R. Baker, "Prioritizing Putative Hydrothermal Sites on Mars" [abstract 1735], in $38^{\text {th }}$ Lunar and Planetary Science Conference Abstracts [CDROM], Lunar and Planetary Institute, Houston, 2007.

47. D. Schulze-Makuch and L.N. Irwin, Life in the Universe: Expectations and Constraints, Springer, 2004.

48. Defense Industry Daily News Release from June 09, 2006: "DARPA's ISIS Project Seeks Slow, Soaring Surveillance Superiority (updated)" Web site: http://www.defenseindustrydaily.com/2006/06/darpas-isis-projectseeks-slow-soaring-surveillance-superiority-updated/index.php

49. DARPA “Integrated Sensor Is Structure" (ISIS) program: http://www.darpa.mil/MTO/programs/isis/index.html

50. Lockheed Martin High Altitude Airship (HAA) program: http://www.lockheedmartin.com/wms/findPage.do?dsp=fec\&ci=14477

51. Defense Industry Daily News Release from January 16, 2006: "Lockheed Wins \$149.2M Contract for High Altitude Airship (updated)" Web site: http://www.defenseindustrydaily.com/2006/01/lockheed-wins-1492m-contract-forhigh-altitude-airship-updated/index.php

52. W. Fink and M.A. Tarbell, "Tier-scalable Reconnaissance Mission Test Bed: Implementation of Ground-Tier" [abstract 2410], in $38^{\text {th }}$ Lunar and Planetary Science Conference Abstracts [CD-ROM], Lunar and Planetary Institute, Houston, 2007. 